\title{
Crianza de animales domésticos como factor de riesgo de fascioliasis humana
}

\author{
Breeding of domestic animals as a risk factor for human fascioliasis
}

Aldo Valderrama P. ${ }^{1,4}$, David Serrano R. ${ }^{1}$, Julio Trujillo C. ${ }^{1}$, Katty Merino T. ${ }^{1}$, Karol Serrano O. ${ }^{1}$, César Gavidia C. ${ }^{2}$, William Quispe P. ${ }^{3}$

\section{Resumen}

El estudio tuvo como objetivo determinar la asociación de fascioliasis con la crianza de animales, características de la vivienda y consumo de alimentos de niños del distrito de Santa María de Chicmo, Apurímac, Perú. Se tomaron muestras serológicas a 493 niños de 6 a 16 años, de ambos sexos, con el consentimiento informado de sus padres. La prevalencia, determinada mediante la técnica de inmunoblot fue de $5.3 \%$ (IC95\% = $3.2 \%-7.4 \% ; 26 / 493)$. El análisis univariado demostró que la crianza de bovinos y gatos, así como la crianza de tres o más cerdos fueron factores asociados a fascioliasis. La crianza de más de cinco ovinos configuró un factor de protección. La fascioliasis en niños no estuvo asociada a las características de las viviendas ni al consumo de alimentos. Se concluye que el distrito de Santa María de Chicmo es mesoendémico para fascioliasis en niños, y que la crianza de algunas especies de animales domésticos implica riesgo de fascioliasis humana.

Palabras clave: fascioliasis; niños; animales domésticos; vivienda; consumo de alimentos

\section{AbSTRACT}

The aim of this study was to determine the association of fascioliasis with the breeding of animals, housing characteristics and food consumption of children in the district of Santa María de Chicmo, Apurimac, Peru. Serological samples were taken from 493 children aged 6 to 16 years, males and females, with the informed consent of their parents. The

\footnotetext{
${ }^{1}$ Facultad de Medicina Veterinaria y Zootecnia, Universidad Nacional Micaela Bastidas de Apurímac - UNAMBA, Abancay, Perú

${ }^{2}$ Universidad Nacional Mayor de San Marcos - UNSMS, Lima, Perú

${ }^{3}$ Instituto Nacional de Salud - INS, Lima, Perú

${ }^{4}$ E-mail: alimvalderrama@gmail.com
}

Recibido: 21 de agosto de 2018

Aceptado para publicación: 20 de febrero de 2019 
prevalence, determined by the immunoblot technique was $5.3 \%(95 \% \mathrm{CI}=3.2 \%-7.4 \%$, 26/493). The univariate analysis showed that the breeding of cattle and cats, and raising three or more pigs were factors associated with fascioliasis. The breeding of more than five sheep resulted as a protection factor. Fascioliasis in children was not associated with the characteristics of the dwellings or the consumption of food. It is concluded that the district of Santa María de Chicmo is mesoendemic for fascioliasis in children, and that the rearing of some species of domestic animals involves risk of human fascioliasis.

Key words: fascioliasis; children; breeding of domestic animals; housing; food consumption

\section{INTRODUCCIÓN}

Fasciola hepatica es un trematodo hermafrodita que causa fascioliasis y afecta a animales herbívoros, omnívoros y ocasionalmente al ser humano (Natividad y Terashima, 2008). Presenta amplia distribución mundial, notificándose desde 1950 en 51 países. Se estima que de 2.4 a 17 millones de personas en el mundo están infectadas y 91 millones están en riesgo de infección, de las cuales, aproximadamente la mitad vive en Bolivia, Ecuador y Perú. La fascioliasis es común en poblaciones de extrema pobreza y viene incrementándose desde 1980 (Marcos et al., 2006). En Perú, las más altas prevalencias en humanos y animales se dan en los valles andinos, hasta los $4500 \mathrm{~m}$, especialmente en las regiones de Arequipa, Cajamarca, La Libertad, Apurímac, Ayacucho, Huánuco, Cuzco, Puno, Piura, Lambayeque y Junín (Jiménez et al., 2011).

Este parásito requiere de reservorio y huésped intermediario para completar su ciclo biológico. Es referida como una trematodiasis propia del ganado bovino y ovino; sin embargo, porcinos y burros han demostrado la capacidad de eliminar y diseminar huevos viables capaces de infectar al molusco limneido. Además, se ha reportado su presencia en equinos, conejos, cuyes, ratones, alpacas, llamas, vicuñas y caprinos (Ayala et al., 2008; Jiménez et al., 2011). Los hospederos definitivos están expuestos a constante reinfección y la falta de un plan de control veterinario permite que se mantenga la endemia. Tal es así que, la convivencia con bovinos, ovinos, llamas y porcinos, entre otros, implicaría riesgo de infección, principalmente en niños (Valencia et al., 2005).

En la región Apurímac no existen estudios de fascioliasis humana, a pesar que presenta características ambientales y sociales similares a las zonas hiperendémicas de Perú, con prevalencias en bovinos y tasas de decomiso de hígados superiores al 80\% (Merino y Valderrama, 2017). La provincia con mayor grado de infección es Andahuaylas, debido a su altitud y a que sus periodos de lluvia coinciden con la estación de verano, donde la población de caracoles y la posibilidad de infección aumentan. A esto se suma la crianza extensiva que favorece a la presencia de la enfermedad, ya que los animales se ponen en contacto directo con la forma infectante del parásito (Valderrama, 2016). Tal es así que $47.6 \%$ de pacientes pediátricos del hospital de Andahuaylas tienen algún parásito con potencial zoonótico (Altamirano et al., 2014). En consecuencia, el objetivo de la investigación fue determinar la seroprevalencia de fascioliasis en niños y establecer si las características de sus viviendas, el consumo de alimentos y la crianza de animales domésticos son factores asociados con la infección parasitaria. 


\section{Materiales y Métodos}

El distrito de Santa María de Chicmo es uno de los 19 distritos de la provincia de Andahuaylas, región Apurímac, Perú. Se encuentra a una altitud de $3272 \mathrm{~m}$ y cuenta con una superficie de $162.1 \mathrm{~km}^{2}$, una población estimada de 9910 habitantes y un nivel de pobreza de $79.4 \%$. El $84 \%$ de la población económicamente activa se dedica a la agricultura y solo 288 viviendas tienen acceso a agua potable de red pública (INEI, 2018). El clima es cálido y templado, con una temperatura media anual de $15.3{ }^{\circ} \mathrm{C}$. La precipitación pluvial media en verano es de $930 \mathrm{~mm}$ y de $10 \mathrm{~mm}$ en invierno (SENAMHI, 2018).

El estudio fue de tipo analítico, transversal, prospectivo y de nivel relacional. Se tomaron muestras de suero sanguíneo de 493 niños de ambos sexos de 6 a 16 años en instituciones educativas. Así mismo, se aplicó una entrevista epidemiológica para identificar las características de su vivienda, consumo de alimentos y crianza de animales domésticos. El tamaño de muestra para determinar la prevalencia de fascioliasis, mediante un muestreo simple al azar, fue de 435 escolares; sin embargo, se logró tomar 493 muestras repartidas proporcionalmente entre la totalidad de las 23 instituciones educativas del distrito estudiado. Así mismo, el tamaño total de la muestra (435) se distribuyó entre dos estratos (I.E. Primaria e I.E. Secundaria), resultando con 209 y 226 muestras, respectivamente. Los niños incluidos en el estudio fueron elegidos aleatoriamente, a quienes se les entregó una ficha para el consentimiento informado del padre o apoderado y una ficha para el asentimiento del niño.

Para todos los procedimientos de laboratorio se siguieron los protocolos del Instituto Nacional de Salud del Ministerio de Salud de Perú (Sánchez et al., 2010, Antitupa et al., 2017).
Para el diagnóstico de fascioliasis se usó la técnica de ELISA-IgG como prueba de tamizaje, para lo cual se emplearon kits (in house) de ELISA-IgG (Sánchez et al., 2010). Se usó el antígeno metabólico «crudo» excretado/secretado de Fasciola hepatica (AMESFH) con una concentración proteica de $1 \mathrm{mg} / \mathrm{ml}$. La sensibilización de la placa de microtitulación se realizó con $100 \mu 1$ de solución de antígeno en cada pozo. Se incubó a $4{ }^{\circ} \mathrm{C}$ durante toda la noche. Posteriormente, se bloquearon los sitios inespecíficos mediante la adición de $100 \mu 1$ de PBS-Tween $0.05 \%$ y leche descremada al 5\%. Se incubó en estufa a $37^{\circ} \mathrm{C}$ por $30 \mathrm{~min}$ y luego se lavaron los pozos adicionando $200 \mu \mathrm{l}$ de PBS-Tween $0.05 \%$. En los pozos se añadieron los sueros control positivo, suero control negativo y sueros problema. Se incubó la placa a $37^{\circ} \mathrm{C}$ por $1 \mathrm{~h}$, descartando el contenido, para luego lavar los pozos. Se colocó $100 \mu 1$ de anti IgG humana peroxidasa HRP diluido 1/1000 para proceder a incubar en estufa a $37^{\circ} \mathrm{C}$ por $1 \mathrm{~h}$. Luego se lavaron los pozos y se colocaron $100 \mu 1$ de la solución de sustrato. Se dejó en oscuridad a temperatura ambiente por $15 \mathrm{~min}$ y se detuvo la reacción adicionando $25 \mu 1$ de ácido sulfúrico $2.5 \mathrm{M}$. La lectura se realizó con el equipo lector de ELISA Biotek ELX800, considerando reactivo (muestras con absorbancias mayores al valor de corte) o no reactivo (muestras con absorbancias iguales o menores al valor de corte). El valor de corte se obtuvo obteniendo el valor promedio de las densidades ópticas de cada placa al cual se le sumó el valor de dos desviaciones estándares.

La técnica de inmunoblot (IB-IgG) se usó como prueba confirmatoria siguiendo el protocolo correspondiente (Sánchez et al., 2010). Se empleó el antígeno metabólico total excretado/secretado de Fasciola hepatica (AMESFH) a una concentración de $2.07 \mu \mathrm{g} / \mu \mathrm{l}$. Para la reacción inmunoenzimática se emplearon placas de plástico divididas en compartimentos y se colocaron tiras de ni- 
trocelulosa conteniendo el antígeno. Se incubaron las tiras en $1 \mathrm{ml}$ de PBS-T conteniendo $5 \%$ de leche descremada (PBS-TL) por 30 min a temperatura ambiente y en agitación. Luego se descartó el PBS-TL, se adicionó $1 \mathrm{ml}$ de los sueros problema diluidos 1:100 (en PBS-TL) e incubó por $1 \mathrm{~h}$ a temperatura ambiente y en agitación. Se lavaron las tiras con PBS-T y se adicionó una solución de anti-IgG humano marcado con peroxidasa diluido a 1:1000 en PBS-TL y se incubó. Se lavaron las tiras con PBS-T y con PBS solo. La reacción se reveló adicionando una solución de $5 \mathrm{mg}$ de DAB, $10 \mu 1 \mathrm{de}$ $\mathrm{H}_{2} \mathrm{O}_{2}(30 \%)$ en $10 \mathrm{ml}$ de PBS. Luego se visualizaron las bandas, se lavaron las tiras con agua deionizada y se dejaron secar a temperatura ambiente en oscuridad. La lectura consistió en visualizar la presencia o ausencia de bandas de precipitación en las tiras de nitrocelulosa. En caso de presencia de bandas, se anotaron sus respectivas masas relativas $(\mathrm{Mr})$ expresadas en kilodaltons $(\mathrm{kDa})$. El criterio de positividad para el diagnóstico es el reconocimiento de uno o más péptidos antígénicos de $\mathrm{Mr}$ entre 17 y $23 \mathrm{KDa}$ (Escalante et al., 2011).

La crianza de animales y las características de la vivienda se determinó realizando una visita a las viviendas de los niños con una guía de observación para identificar factores de riesgo de fascioliasis (Marcos et al., 2004, 2005, 2006; Ayala et al., 2008; Páucar et al., 2010; Rodríguez et al., 2011; Matthys et al., 2011).

El consumo de alimentos se determinó mediante una entrevista epidemiológica para identificar los alimentos consumidos habitualmente que suelen constituir factores de riesgo de fascioliasis (Bendezú et al., 1982; MasComa et al., 2001; Marcos et al., 2006; Dorny et al., 2009; Páucar et al., 2010; Orlando, 2011; León y Cabanillas, 2014; WHO, 2015).

El procesamiento y análisis de datos se realizó con el programa Excel 2010 y el programa SPSS 23. Se compararon estadís- ticamente las variables categóricas con la prueba de Chi cuadrado y Odds ratio (OR) con intervalos de confianza al $95 \%$ y valor de $p<0.05$ como nivel crítico de significancia. Asimismo, se realizó una prueba de regresión logística múltiple para determinar posibles asociaciones a fascioliasis.

El proyecto de investigación fue aprobado por el Instituto Nacional de Salud, el cual incluyó la opinión favorable del Comité Institucional de Ética en Investigación. Las muestras de los estudiantes se tomaron con su asentimiento y el consentimiento informado de los padres o apoderados, quienes firmaron una autorización para que los niños formen parte de la investigación y proporcionen muestras serológicas, además de la entrevista.

\section{Resultados}

La prevalencia de fascioliasis en niños del distrito de Santa María de Chicmo fue de $5.3 \%$ (IC95\%=3.2-7.4\%; 26/493). La edad, sexo e institución educativa no mostraron asociación estadística significativa con fascioliasis. El 99.6\% de estudiantes cría algún animal doméstico en su vivienda (IC95\%=98.5-99.9\%; 491/493). El Cuadro 1 muestra, según el análisis univariado, asociación entre fascioliasis y la crianza de algunos animales domésticos $(p<0.05)$. Los niños que crían bovinos y gatos en sus viviendas tuvieron 4.1 y 2.6 veces más riesgo de presentar fascioliasis que aquellos niños que no crían estas especies, respectivamente.

El Cuadro 2 muestra que la cantidad de ovinos y porcinos criados en las viviendas estuvo asociada a la seroprevalencia de fascioliasis en niños $(p<0.05)$. Tal es así que en las viviendas donde se crían entre 3-5 o más porcinos tuvieron 3.2 y 4.1 veces más riesgo de fascioliasis, respectivamente, que en aquellas donde crían entre 1-2 cerdos. Por otro lado, los niños que crían en sus viviendas más de cinco ovinos tuvieron $90 \%$ más pro- 
Cuadro 1. Crianza de animales domésticos y seroprevalencia de fascioliasis en niños del distrito de Santa María de Chicmo, Andahuaylas, 2017

\begin{tabular}{|c|c|c|c|c|c|c|}
\hline \multirow{2}{*}{$\begin{array}{l}\text { Especie } \\
\text { criada }\end{array}$} & \multicolumn{2}{|c|}{ Con fascioliasis } & \multicolumn{2}{|c|}{ Sin fascioliasis } & \multirow{2}{*}{$\begin{array}{c}\text { OR } \\
\text { (IC95\%) }\end{array}$} & \multirow{2}{*}{$\mathrm{p}$} \\
\hline & $\mathrm{n}$ & $\%$ & $\mathrm{n}$ & $\%$ & & \\
\hline Gato & 7 & 10.6 & 59 & 89.4 & $2.6(1.0-6.3)$ & 0.044 \\
\hline Pato & 11 & 6.9 & 148 & 93.1 & $1.6(0.8-3.5)$ & 0.263 \\
\hline Ovino & 16 & 6.9 & 216 & 93.1 & $1.9(0.8-4.2)$ & 0.134 \\
\hline Bovino & 23 & 7.0 & 305 & 93.0 & $4.1(1.2-13.8)$ & 0.024 \\
\hline Perro & 24 & 6.3 & 354 & 93.7 & $3.8(0.9-16.5)$ & 0.071 \\
\hline Gallina & 23 & 5.9 & 368 & 94.1 & $2.1(0.6-7.0)$ & 0.246 \\
\hline Cerdo & 25 & 6.0 & 391 & 94.0 & $4.9(0.7-36.4)$ & 0.124 \\
\hline Cuy & 26 & 5.6 & 435 & 94.4 & - & 0.998 \\
\hline Otros $^{1}$ & 35 & 4.1 & 70 & 95.9 & - & 0.630 \\
\hline
\end{tabular}

${ }^{1}$ Pavo, caballo, conejo

Cuadro 2. Cantidad de animales domésticos criados en viviendas y seroprevalencia positiva de fascioliasis en niños del distrito de Santa María de Chicmo, Andahuaylas, 2017

\begin{tabular}{lcccc}
\hline \multirow{2}{*}{ Especie criada } & \multicolumn{3}{c}{$\begin{array}{c}\text { Niños con fascioliasis } \\
\text { n (\%) }\end{array}$} & \multirow{2}{*}{ Total (n) } \\
\cline { 2 - 4 } & $1-2$ animales & $3-5$ animales & $>5$ animales & \\
\hline Perro & $23(95.8)$ & $1(4.2)$ & - & 24 \\
Gato & $7(100.0)$ & - & - & 7 \\
Gallina & $2(8.7)$ & $6(26.1)$ & $15(65.2)$ & 23 \\
Pato & $2(25.0)$ & $3(37.5)$ & $3(37.5)$ & 8 \\
Cuy & - & $1(4.2)$ & $23(95.8)$ & 24 \\
Ovino & $13(81.3)$ & $1(6.3)$ & $\mathrm{a} 2(12.5)$ & 16 \\
Bovino & $9(39.1)$ & $11(47.8)$ & $3(13.0)$ & 23 \\
Porcino & $13(52.0)$ & $\mathrm{b} 6(24.0)$ & $\mathrm{c} 6(24.0)$ & 25 \\
Otros & $4(100.0)$ & - & - & 4 \\
\hline
\end{tabular}

a $\mathrm{p}=0.010 ; \mathrm{OR}=0.1 ; \mathrm{IC} 95 \%=0.0-0.6$

${ }^{b} p=0.025 ; O R=3.2 ; I C 95 \%=1.2-8.8$

${ }^{c} \mathrm{p}=0.007 ; \mathrm{OR}=4.1 ; \mathrm{IC} 95 \%=1.5-11.5$ 
Cuadro 3. Características de la vivienda y seroprevalencia de fascioliasis en niños del distrito de Santa María de Chicmo, Andahuaylas, 2017

\begin{tabular}{lcccc}
\hline $\begin{array}{l}\text { Características de la } \\
\text { vivienda }\end{array}$ & $\begin{array}{c}\text { Niños con } \\
\text { fascioliasis } \\
\mathrm{n}(\%)\end{array}$ & $\begin{array}{c}\text { Niños sin } \\
\text { fascioliasis } \\
\mathrm{n}(\%)\end{array}$ & $\begin{array}{c}\text { OR } \\
(\mathrm{IC} 95 \%)\end{array}$ & $\mathrm{p}$ \\
\hline $\begin{array}{l}\text { Defecan a campo } \\
\text { abierto }\end{array}$ & - & $39(100.0)$ & - & 0.998 \\
$\begin{array}{c}\text { Disponen excretas en } \\
\quad \text { letrina }\end{array}$ & - & $25(100.0)$ & - & 0.998 \\
$\begin{array}{c}\text { Presencia de actividad } \\
\text { agrícola }\end{array}$ & $25(5.5)$ & $433(94.5)$ & $1.9(0.3-14.9)$ & 0.515 \\
$\begin{array}{c}\text { Suministro de agua por } \\
\text { canales }\end{array}$ & $25(5.4)$ & $436(94.6)$ & $1.8(0.2-13.6)$ & 0.579 \\
$\begin{array}{c}\text { Tiene piso de tierra } \\
\text { Tiene una habitación en } \\
\quad \text { la vivienda }\end{array}$ & $24(5.9)$ & $386(94.1)$ & $2.5(0.6-10.9)$ & 0.216 \\
$\begin{array}{c}\text { Ubicada al borde de } \\
\text { acequia }\end{array}$ & $6(8.5)$ & $65(91.5)$ & $1.9(0.7-4.8)$ & 0.202 \\
$\begin{array}{c}\text { Ubicada al borde de } \\
\text { canal de regadío }\end{array}$ & $23(6.4)$ & $338(93.6)$ & $2.9(0.9-9.9)$ & 0.085 \\
$\begin{array}{l}\text { Ubicada al borde de } \\
\text { cultivos }\end{array}$ & $23(5.8)$ & $376(94.2)$ & $1.9(0.6-6.3)$ & 0.323 \\
\hline
\end{tabular}

Cuadro 4. Consumo de alimentos y seroprevalencia de fascioliasis en niños del distrito de Santa María de Chicmo, Andahuaylas, 2017

\begin{tabular}{lcccc}
\hline Consumo de alimentos & $\begin{array}{c}\text { Niños con } \\
\text { fascioliasis } \\
\mathrm{n}(\%)\end{array}$ & $\begin{array}{c}\text { Niños sin } \\
\text { fascioliasis } \\
\mathrm{n}(\%)\end{array}$ & $\begin{array}{c}\text { OR } \\
\text { (IC95\%) }\end{array}$ & $\mathrm{p}$ \\
\hline Agua no tratada & $24(5.5)$ & $414(94.5)$ & $1.5(0.4-6.7)$ & 0.567 \\
Alfalfa & $14(5.8)$ & $228(94.2)$ & $1.2(0.6-2.7)$ & 0.618 \\
Arroz & $26(5.4)$ & $457(94.6)$ & - & 0.999 \\
Berro & $24(5.4)$ & $420(94.1)$ & $1.3(0.3-5.9)$ & 0.695 \\
Brócoli & $24(5.8)$ & $390(94.2)$ & $2.4(0.6-10.2)$ & 0.248 \\
Cebolla cruda & $26(5.4)$ & $453(94.6)$ & - & 0.999 \\
Emolientes & $10(5.0)$ & $192(95.0)$ & $0.9(0.4-2.0)$ & 0.789 \\
Ensaladas & $26(5.4)$ & $459(94.6)$ & - & 0.999 \\
Espinaca & $25(5.7)$ & $410(94.3)$ & $3.5(0.5-26.2)$ & 0.226 \\
Hierbabuena & $22(5.3)$ & $395(94.7)$ & $1.0(0.3-2.9)$ & 0.996 \\
Lechuga & $26(5.3)$ & $461(94.7)$ & - & 0.999 \\
Maíz & $25(5.4)$ & $438(94.6)$ & $1.7(0.2-12.7)$ & 0.627 \\
Rábano & $19(5.6)$ & $318(94.4)$ & $1.3(0.5-3.1)$ & 0.596 \\
Plantas acuáticas & $14(4.9)$ & $274(95.1)$ & $0.8(0.4-1.8)$ & 0.627 \\
Verduras crudas & $26(5.3)$ & $462(94.7)$ & - & 0.999 \\
\hline
\end{tabular}


tección contra fascioliasis que los niños que crían entre 1-2 ovinos. El análisis de regresión logística multivariado mostró que la crianza de bovinos estuvo asociada a fascioliasis $(\mathrm{OR}=4.2 ; \mathrm{IC} 95 \%=1.2-14.5 ; \mathrm{p}=0.023)$.

Por otro lado, se determinó que no existió asociación estadística significativa entre el sexo de animales domésticos criados en las viviendas y la seroprevalencia de fascioliasis en niños. Así mismo, la crianza de animales domésticos (mascotas o animales de crianza extensiva), dentro o fuera de la vivienda, tampoco estuvo asociada a la seroprevalencia de fascioliasis.

El 93.5\% de las viviendas tienen suministro de agua por canales de regadío $($ IC95\%=91.2-95.8; 461/493), 92.9\% tienen presencia de actividad agrícola (IC95\%=90.5-95.3; 458/493), 83.2\% tienen piso de tierra $($ IC95\%=79.8-86.6; 410/493) y $80.9 \%$ están ubicadas al borde de cultivos (IC95\%=77.4-84.5; 399/493). Las demás características tuvieron una ocurrencia menor del $80 \%$. No se encontró asociación significativa entre la fascioliasis en niños y las características de sus viviendas (Cuadro 3).

Se encontró que $98.9 \%$ de los niños consumen habitualmente verduras crudas (IC95\% $=97.7-99.7 ;$ 488/493), 98.8\% consumen lechugas (IC95\%=97.7-99.9; 487/493), $98.4 \%$ ensaladas (IC95\% $=97.2 / 99.6 ; 485 / 493$ ), $97.8 \%$ arroz (IC95\% $=96.6-99.3 ; 483 / 493$ ), $97.2 \%$ cebolla cruda (IC95\% $=95.6-98.7 ; 479$ / 493), 93.9\% mazorca de maíz (IC95\% $=91.7$ $96.1 ; 463 / 493$ ) y $90.1 \%$ berro (IC95\% $=87.3$ $92.8 ; 444 / 493)$. Los demás alimentos son consumidos en menor proporción. No existió asociación significativa entre la seroprevalencia de fascioliasis en niños y el consumo de determinado tipo de alimentos (Cuadro 4).

\section{Discusión}

La prevalencia de fascioliasis encontrada en el presente estudio clasifica al distrito- de Santa María de Chicmo como mesoendémico, debido a que se encuentra entre $1 \mathrm{y}$ 5\% (Jiménez et al., 2011), al igual que otras zonas de Perú como Huancabamba, Arequipa, Chontabamba, Celendín, Huancavelica, San Juan, Baños del Inca y Caujul (Albán et al., 2002; Marcos et al., 2006; Ayala et al., 2008; León y Cabanillas, 2014; Antitupa et al., 2017). En el Perú, las regiones endémicas se encuentran en zonas altoandinas, debido a que los caracoles limneidos infectados sobreviven más tiempo, ya que el periodo de eliminación de cercarias es más largo y el número de metacercarias producidas por el caracol es mayor (Marcos et al., 2006). Las localidades rurales más altas y alejadas de las ciudades, y con difícil acceso por carreteras, pueden tener mayor riesgo de infección humana. Por otro lado, la prevalencia hallada en este estudio es similar a lo reportado en zonas mesoendémicas de los Andes ecuatorianos (Marcos et al., 2005), Cuba (Marcos et al., 2004), Kirguistán (Marcos, 2012) y algunas localidades venezolanas (Mas-Coma et al., 1999), mexicanas (Mas-Coma et al., 2001) y de Tayikistán (Matthys et al., 2011).

La investigación no mostró diferencia estadística significativa entre grupos de edad. Los brotes suelen afectar a pocos individuos, los cuales resultan infectados a partir de la misma fuente de contaminación (familia o pequeño grupo, consumo de berros u otras hortalizas de cultivo silvestre o comercializadas, portadoras de metacercarias) (Jiménez et al., 2011). Del mismo modo, no se encontró diferencia entre la prevalencia de instituciones educativas de primaria y secundaria, al igual que en otras investigaciones (Altamirano et al., 2014; Arlett y Pérez, 2007), considerando que la población humana de edad escolar es la que presenta mayor riesgo de infección por fascioliasis (Merino y Valderrama, 2017, Marcos et al., 2006), no necesariamente por ser la población más susceptible al deficiente sistema sanitario de las zonas endémicas, sino por la deficiente inmunidad que existe en esta población para evitar la infección (Merino y Valderrama, 2017). 
Por otro lado, esta investigación tampoco mostró diferencias significativas en la prevalencia entre varones y mujeres, debido a que en Santa María de Chicmo las labores de campo son compartidas entre varones y mujeres; del mismo modo, se tiene una participación igualitaria en los centros educativos (Natividad y Terashima, 2008; Altamirano et al., 2014; Arlett y Pérez, 2007). No obstante, un estudio en Ecuador reporta predominio del sexo femenino en pacientes con fascioliasis (Orlando, 2011).

Las diferentes prevalencias de fascioliasis entre instituciones educativas podrían deberse a que aquellas más alejadas del centro poblado tienen menor accesibilidad a servicios básicos como agua y alumbrado eléctrico (Natividad et al., 2008). De la misma forma, la mayor cercanía a acequias podría aumentar la probabilidad de infección debido al contacto con aguas contaminadas con presencia de la metacercarias viables (Natividad y Terashima, 2008). Asimismo, la eliminación de excretas en silos constituye un factor protector para tener serología positiva, posiblemente debido a que estos niños tienen mejores hábitos higiénicos que aquellos que eliminan sus heces en el campo, río o acequias.

Se encontró asociación entre fascioliasis y la crianza de bovinos y gatos en las viviendas. Esta parasitosis requiere de un reservorio y un huésped intermediario para completar su ciclo biológico y usualmente es referida como una trematodiasis propia del ganado bovino; sin embargo, parasita diversas especies. Tal es así que, la convivencia con animales implica riesgo de infección, principalmente en niños (Ayala et al., 2008). En la sierra peruana existe una estrecha convivencia con herbívoros rumiantes y otros mamíferos que genera riesgos para la salud de la familia (Santa María, 2008; Dorny et al., 2009).

La crianza de más de cinco ovinos constituyó un factor de protección contra fascioliasis, al igual que un estudio previo
(Páucar et al., 2010), debido posiblemente a que la tenencia de muchas ovejas puede estar aunada con el tratamiento antihelmíntico. Por otro lado, la investigación mostró que los niños que crían entre 3-5 o más cerdos tienen 3.2 y 4.1 veces más riesgo de fascioliasis, respectivamente, que los niños que crían entre 1-2 cerdos. La mayor cantidad de cerdos incrementaría el riesgo de diseminación de fascioliasis debido a su hábito de consumir todo tipo de desechos, heces y a su crianza extensiva (Marcos et al., 2006).

La investigación mostró que no existió asociación estadística significativa entre el sexo de animales domésticos criados en las viviendas y la seroprevalencia de fascioliasis en niños, a pesar de que predomina la cantidad de hembras (por ejemplo, un carnero por 45 ovejas [Rodríguez et al., 2011], 3-7 toros por 100 vacas [Sánchez et al., 2010]).

\section{Conclusiones}

- $\quad$ El distrito de Santa María de Chicmo es mesoendémico para fascioliasis en niños.

- La crianza de bovinos y gatos en las viviendas de los niños constituye un factor de riesgo de fascioliasis, al igual que criar tres o más cerdos. La crianza de más de cinco ovinos configura un factor de protección.

- La fascioliasis en niños no estuvo asociada a características de las viviendas ni al consumo de alimentos.

- De acuerdo con el análisis multivariado, la crianza de bovinos constituye un factor de riesgo de fascioliasis.

\section{Literatura Citada}

1. Albán M, Jave J, Quispe T. 2002. Fasciolasis en Cajamarca. Rev Gastroenterol Perú 22: 28-32.

2. Altamirano F, López $R$, Puray $N$. 2014. Enteroparásitos con potencial zoonótico en pacientes pediátricos del 
Hospital de Andahuaylas - Apurímac. Salud Tecnol Vet 2: 14-19.

3. Antitupa I, Quispe W, Mayo J, Valverde F, Sánchez E. 2017. Inmunoblot-IgG con antígenos semipurificados (27-28KDA) para el diagnóstico confirmatorio de fasciolosis humana. Rev Perú Med Exp Salud Pública_34: 41-42.

4. Arlett M, Pérez M. 2007. Fasciola hepatica en Venezuela: revisión histórica. Rev Fac Cs Vet UCV 48: 3-14.

5. Ayala MS, Bustamante ES, González M. 2008. Estado actual de la Fascioliasis en Mollebamba, Santiago de Chuco, Región La Libertad y su abordaje por niveles de atención y prevención. Rev Med Vallejiana 5: 90-99.

6. Bendezú P, Frame A, Hillyer GV. 1982. Human fascioliasis in Corozal, Puerto Rico. J Parasitol 68: 297-299. doi: $10.2307 / 3281190$

7. Díaz, Rarcés M, Millán LM, Pérez J, Millán JC. 2011. Comportamiento clínico-terapéutico de Fasciola hepatica en una serie de 87 pacientes. Rev Cubana Med Trop 21: 268-274.

8. Dorny P, Praet N, Deckers N, Gabriel S. 2009. Emerging food-borne parasites. Vet Parasitol 163: 196-206. 196-206. doi: 10.1016/j.vetpar.2009.05.026

9. Escalante H, Davelois K, Ortiz P, Rodríguez H, Díaz E, Jara C. 2011. Estandarización de la técnica de western blot para el diagnóstico de la fasciolosis humana utilizando antígenos de excreción-secreción de Fasciola hepatica. Rev Peru Med Exp Salud Pública 28: 454-461. doi: 10.17843/rpmesp.2011.283.523

10. [INEI] Instituto Nacional de Estadística e Informática. 2018. Perú en cifras. [Internet]. Disponible en: http:// www.inei.gob.pe/

11. Jiménez J, Vergel K, Velásquez $M$, Vega F, Uscata R, Romero S, Flórez $A$, et al. 2011. Parasitosis en niños en edad escolar: relación con el grado de nutrición y aprendizaje. Rev Horizonte Médico 11: 65-69.

12. León D, Cabanillas O. 2014. Factores de riesgo asociados a fasciolosis humana en tres provincias del departamento de Cajamarca, Perú (Periodo 2010). Salud Tecnol Vet 2: 7-13. doi: 10.20453/ stv.v2i1.2061

13. Marcos LA. 2012. An overview of fasciolosis: epidemiology, clinical manifestations, diagnosis and treatment. Per $\mathrm{J}$ Parasitol 20:

14. Marcos LA, Maco V, Terashima A, Samalvides F, Miranda E, Tantalean M, Espinoza JR, et al. 2004. Hiperendemicidad de fasciolosis humana en el valle del Mantaro, Perú: factores de riesgo de la infección por Fasciola hepatica. Rev Gastroenterol Perú 24: 158-164.

15. Marcos L, Maco V, Terashima A, Samalvides F, Espinoza JR, Gotuzzo E. 2005. Fascioliasis in relatives of patients with Fasciola hepatica infection in Peru. Rev Inst Med Trop SP 47: $219-$ 222. doi: 10.1590/S0036-46652005000400008

16. Marcos L, Maco V, Samalvidesa F, Terashima A, Espinoza JR, Gotuzzo E. 2006. Risk factors for Fasciola hepatica infection in children: a casecontrol study. T Roy Soc Trop Med H 100: 158-166. doi: 10.1016/j.trstmh.2005.05.016

17. Mas-Coma S, Esteban JG, Bargues MD. 1999. Epidemiology of human fascioliasis: a review and proposed new classification. B World Health Organ 77: 340-346.

18. Mas-Coma S, Funatsu IR, Bargues MD. 2001. Fasciola hepatica and lymnaeid snails occurring at very high altitude in South America. Parasitology 123: 115-127. doi: 10.1017/S0031182001008034

19. Matthys B, Bobieva M, Karimova $G$, Mengliboeva $Z$, Jean-Richard V, Hoimnazarova M, Kurbonova M, et al. 2011. Prevalence and risk factors of helminths and intestinal protozoa 
infections among children from primary schools in western Tajikistan. Parasite Vector 4: 195. doi: 10.1186/1756-33054-195

20. Merino K, Valderrama AA. 2017. Fasciola hepatica en bovinos del valle interandino de Aymaraes (Perú): identificación de factores asociados. Rev Med Vet 34: 137-147. doi: 10.19052/mv.4262

21. Natividad IS, Terashima A. 2008. Prevalencia de infección humana por Fasciola hepatica en pobladores del distrito de Caujul provincia de Oyon, región de Lima, Perú. Acta Med Peru 25: 77-80.

22. Orlando A. 2011. Prevalencia y factores asociados a la Fasciola hepatica y otras parasitosis intestinales en la comunidad de Tarqui - 2011. Tesis de Maestría. La Habana, Cuba: Instituto de Medicina Tropical «Pedro Kourí». 41 p.

23. Páucar S, Chávez A, Casas E, Suárez F. 2010. Prevalencia de fasciolasis y paramfistomiasis en el ganado lechero de Oxapampa, Pasco. Rev Inv Vet Perú 21:87-92. 10.15381/rivep.v21i1.314

24. Rodríguez, C, Rivera M, Cabanillas $Q$, Pérez M, Blanco H, Gabriel J, Suarez $W$. 2011. Prevalencia y factores de riesgo asociados a parasitosis intestinal en escolares del distrito de Los Baños del
Inca, Perú. UCV-Scientia 3: 181-186

25. Sánchez EL, Náquira CG, Vega ES, Miranda EF, Quispe WM, Ayala ER. 2010. Manual de procedimientos para el diagnóstico serológico de las zoonosis parasitarias. Serie de Normas Técnicas No. $32.2^{\circ}$ ed. Lima: Ministerio de Salud. $106 \mathrm{p}$.

26. Santa María R. 2008. La iniciativa de vivienda saludable en el Perú. Rev Peru Med Exp Salud Publica 25: 419-430.

27. [SENAMHI] Servicio Nacional de Meteorología e Hidrología del Perú. 2018. Perú: clima en el Perú. [Internet]. Disponible en: http://www.senamhi.gob.pe/

28. Valderrama AA. 2016. Prevalencia de fascioliasis en animales poligástricos de Perú, 1985-2015. Rev Med Vet 32: 121129. doi: $10.19052 / \mathrm{mv} .3861$

29. Valencia N, Pariona A, Huamán M, Miranda F, Quintanilla S, Gonzáles A. 2005. Seroprevalencia de fasciolosis en escolares y en ganado vacuno en la provincia de Huancavelica, Perú. Rev Peru Med Exp Salud Pública 22: 96-102.

30. [WHO] World Health Organization. 2015. Foodborne trematode infections. [Internet]. Disponible en: http:// www. who.int/foodborne trematode_infections/fascioliasis/en/\# 